DE LA

\section{SOCIÉTÉ BOTANIQUE}

DE FRANCE

\section{Tome 139}

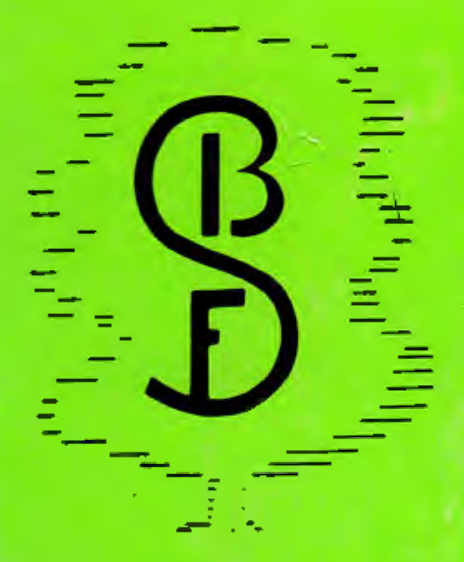

$$
1992-2 / 3 / 4
$$

\section{LES CHARBONS DE BOIS} LES ANCIENS ÉCOSYSTĖMES ET LE RÔLE DE L'HOMME

Colloque organisé à Montpellier du 10 au 13 septembre 1991

$$
\begin{gathered}
\text { par } \\
\text { J.-L. VERNET }
\end{gathered}
$$


Bull. Soc. bot. Fr., 139, Actual. bot. (2/3/4), 597-608, (1992).

\title{
Anthracologie et espaces forestiers charbonnés. Quelques exemples dans la moitié orientale des Pyrénées
}

\author{
par Bernard DAVASSE
}

\begin{abstract}
CIMA-URA 366/CNRS, Institut de Géographie, Universicé Toulouse-Mirail, F-31058 Toulouse
\end{abstract}

Résumé. Les chaßbonnières tèmoignent de l'exploilation intenslve des forêts pour alimenter la metallurgie au bols. Ces restes d'une actlvité proto-industrielle quil a duré pendant toute la pérlode historlque se rencontrent un peu partout dans la mohié orientale des Pyréneses. Grace a l'anthracologie, les charbonnisres devlennent un moyen heurlsitiqu permettant de restituer dans le temps les espaces forestiers.

Une mithodologle specifique, adapiée à l'objet (la charbonnlère) et a la problématique (la blog6ographle), amene a distinguer trols niveaux d'analyse :

1.- L'espece biologique. C'est a ce premler niveau que se pose le problame de la détermination anatomlque du pin sylvestre dans les hautes vallees des Pyrénees artegeolses ;

2.- Les successions forestlares sur le site charbonné (espace hormogène, tant d'un polnt de vue biogéographique que topoclimatlque, où sont étudieses plusieurs charbonnißres) ;

3.- L'evolutlon de l'espace forestler a l'échelle d'une vallee. La répartitlon des charbonnieres, l'étude de sites charbonnés ́chelonnes selon un gradlent althudinal et les datatlons au Carbone 14 permettent de reconstituer cette 6 volution.

L'appilcation de l'étude des charbonnieres à des vallés de la moltíe orientale des Pyrénées que sont le Vicdessos et lAston (Arlage, France) illustre la démarche, et confirme sa valldité. On obtlent alors un éclairage nouveau sur l'organisation écologlque de la végetation qui se révele etre une confrontation permanente entre les processus naturels et le poids des gestions de l'espace par les soclétés.

Summary.- The charcoal kilns are surviving remains of forestry exploltation by wood motalworking. These proto-industrial deposils can be found in the whole eastern Pyrenees. The anthracology mathod allows us to recreate the forest landscape in space and time.

A specific methodology which applies both to the subject (charcoal kiln) and the problem (biogeography) has enabled us to dlstingulsh three levels of analysts.

1.- The specles. Al this first level, the problem of the anatomic determination of scots pine (Pinus sylvestris L.) In the upper valleys of Artege Pyrenees must be conskered.

2.- The plant successions in the charcoal kilns spot which is an homogeneous space as much from a biogeographical point of vlew as from a topoclimatical one.

3.- The evolution of the forest landscape in a valley. It can be reconstituted thanks to the charcoal kilns distribution, the antracology analysis and radlocaron dates.

The charcoal kilns study has been put Into practice In eastem Pyrenees valleys : Vlcosssos and Aston (Arlege, France). The results suggest a new interpretation of ecological organisation of vegetation which Is a combinatlon between natural processes and socleties managements.

French Pyrenees.

Key words : historical ecology - charcoal analysis - forest - charcoal kilns - biogeography - 
Les charbonnières sont le témoignage d'une exploitation intensive dea forêts liée aux besoins énergétiques des activités proto-industrielles. Entre k Moyen Age, voire l'époque gallo-romaine, et la fin du XIXème siècle, une importante métallurgie au bois s'est développée dans la moitié orientale des Pyrénées. Elle a été un des principaux facteurs de transformation de l'espace montagnard. Grâce à l'anthracologie, les charbonnières deviennent un moyen heuristique permettant de restituer dans la longue durée historique l'évolution des espaces forestiers.

Toute recherche anthracologique se doit d'étudier les pratiques et les techniques d'exploitation du milieu par l'homme. L'étude anthracologique des charbonnières n'échappe pas à cette règle. Mais, dans les Pyrénées, le charbonnage ne semblant pas avoir retenu l'attention de ses contemporains, la doctmentation disponible reste rare. C'est donc principalement sur quelques témoignages récents (Izard, à paraître) et sur les relevés de terrains que l'étude peut s'appuyer. Le charbon de bois était transformé sur les lieux mêmes de l'abattage (meules de 10 à 15 stères en général au milieu du XIXème siècle). Lo charbonnière est de ce fait le reflet d'un prélèvement localisé (étude spatiale), dont l'étendue est déterminée par les conditions topographiques (sur versant abrupt par exemple, le dénivelé drainé par l'exploitation peut dépasser la centaine de mètres). La place de charbonnage était dans la plupart des cas régulic̀rement réutilisée, on peut donc observer la succession des peuplements charbonnés (étude dynamique). Le défournement de la charge, une fois celle-ci refroidie, s'effectuait par "ratissage", il y a donc homogénéisation spatiale des charbons de bois sur le site. Parfois on mettait en œuvre de véritables "chantiers de charbonnage", aménageant plusieurs charbonnières à proximité les unes des autres, vraisemblablement par contrainte topographique ou pour faciliter le travail (surveillance aisée d'un ensemble de charbonnières à différentes étapes de transformation). Lorsqu'on est en présence d'un tel système, il est possible de retrouver la (ou les) période(s) synchrone(s) aux différentes charbonnières.

C'est en tenant compte de ces observations, aussi bien que des acquis de l'anthracologie archéologique, qu'après plusieurs tâtonnements une méthode d'étude des replats de charbonnage a été mise au point. Adaptée à l'objet (la charbonnière) et à la problématique (la biogéographie), la méthode que nous avons appliquée est celle du site charbonné. Elle consiste en l'étude de plasieurs charbonnières dans un espace homogène tant d'un point de vue biogéographique que topoclimatique (Davasse, 1992). Au niveau de chaque charbonnière, le prélèvement des charbons de bois s'effectue de la manière suivante : au point le plus profond de la charbonnière, un carré d'un quart $\mathrm{de} \mathrm{m}^{2}$ est tracé ; après enlèvement de l'horizon supérieur stérile, le sédiment charbonneux est prélevé par décapages successifs de $5 \mathrm{~cm}$, jusqu'à l'horizon inférieur marqué par un changement net de couleur ; ensuite, en évitant si possible le fractionnement, le sédiment est tamisé à l'eau à la maille de $5 \mathrm{~mm}$; on récupère alors les fragments de charbon de bois. Après analyse d'un nombre optimal de fragments (100 à 180), la comparaison des proportions des taxons d'un décapage à l'autre autorise une interprétation dynamique. Ces variations sont consignées dans le diagramme anthracologique construit pour chaque site.

Un premier bilan de l'étude anthracologique des restes de charbon- 
nage dane la moitié orientale des Pyrénées peut montrer l'intérêt et les problèmes de l'interprétation des données à trois niveaux différents d'analyse : celui de l'espèce biologique, qui soulève le problème posé par la détermination anatomique du pin sylvestre dans la Haute-Ariège, celui des successions forestières sur le site charbonné et enfin celui de l'évolution de l'espace forestier valléen.

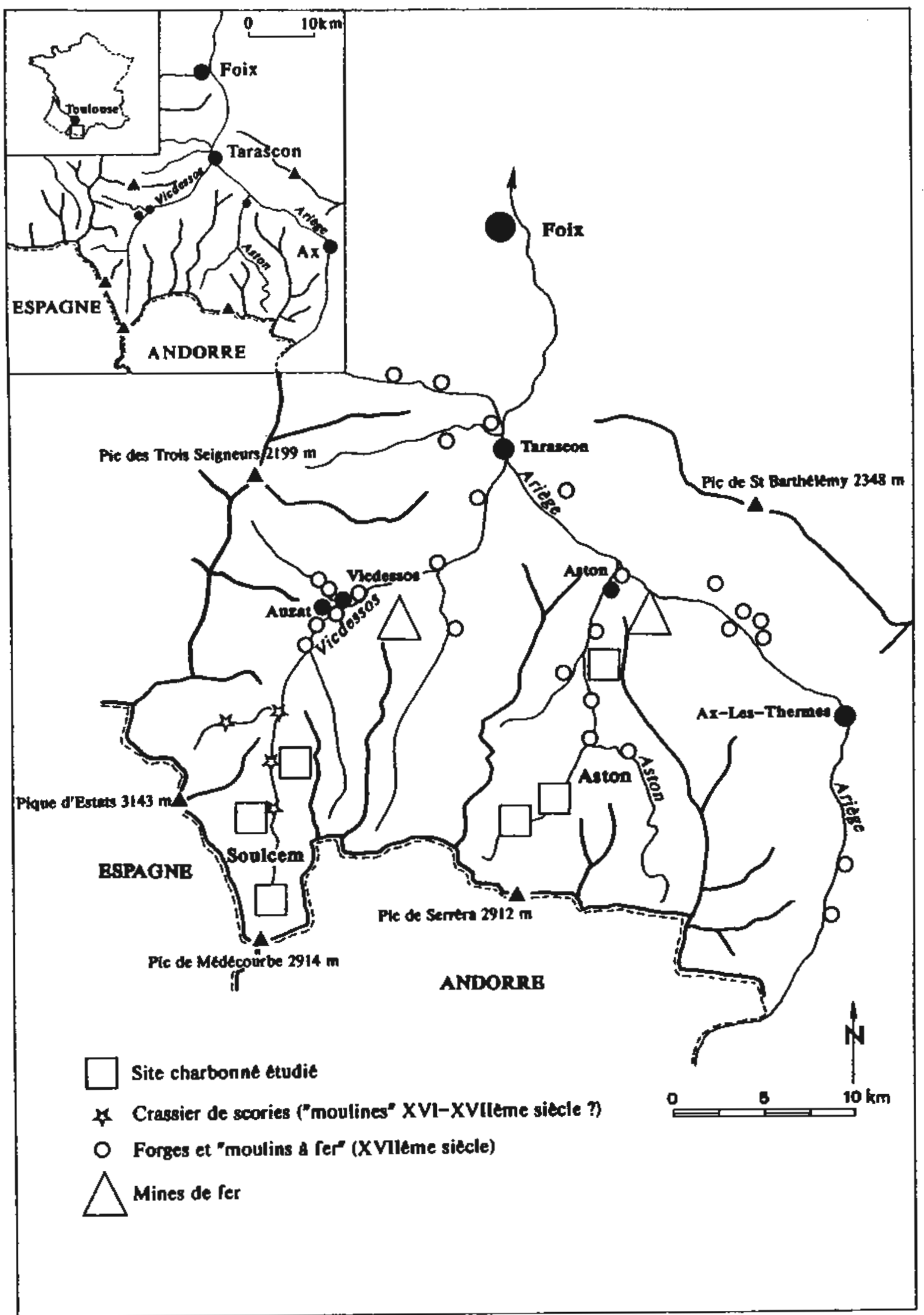




\section{1 - LE PIN SYLVESTRE AUX PYRENEES ARIEGEOISES : DU PROBLEME DE LA DETERMINATION ANATOMIQUE A L'ANALYSE BIOGEOGRAPHIQUE}

Jusqu'à ce jour, un des apports de l'anthracologie des charbonnières était la mise en évidence de la présence passée importante du pin sylvestre dans les Pyrénées ariégeoises - phénomène non perçu par les naturalistes - alors qu'il n'y existe pas actuellement. On déterminait en effet fréquemment cette essence dans les replats de charbonnage, entre 1400 et $2000 \mathrm{~m}$ d'altitude (Bonhôte et Vernet, 1988 ; Davasse et Galop, 1989, 1990 ; Davasse, 1992). Ces résultats s'appuyaient sur une détermination spécifique, en coupe transversale, du pin sylvestre et du pin à crochets (Pinus uncinata Ram.), anatomiquement proche. Pour le pin à crochets, les critères retenus étaient les suivants : accroissements annuels très fins, bois d'été réduit, passage brusque du bois initial au bois final ; pour le pin sylvestre : passage progressif du bois initial au bois final, bois final développé où se répartissaient les canaux sécréteurs (Jacquiot, 1955, Heinz et al., 1990). Or, il s'est avéré que l'utilisation de ces critères lors de l'étude des charbonnières donnait certains résultats aberrants. Ce qui nous a amené à remettre en question le validité de ces déterminations. Etaient-elles bien inhérentes à l'anatomie du bois ou rendaient-elles compte uniquement d'une adaptation à des facteurs bioclimatiques, une différence d'implantation pouvant se manifester aux niveaux des anneaux de croissance ? Il a donc paru nécessaire d'étudier le comportement anatomique des deux pins lorsqu'ils sont à la même altitude et dans les mêmes conditions stationnelles. Une vingtaine d'échantillons de troncs actuels ont été carbonisés en laboratoire. Ils provenaient de diverses stations des Pyrénées ariégeoises (Aston et Vicdessos) mais aussi des Pyrénées orientales (Osséja) et des Pyrénées catalanes espagnoles (Vallferrera). Toutes étaient situés entre 1400 et $1800 \mathrm{~m}$, dans des secteurs où les deux pins cohabitent. Cette étude sommaire a mis en évidence la précarité des critères anciennement utilisés, qui semblent effectivement être liés au bioclimat de la station et/ou à la densité du peuplement. De nouveaux critères n'ont pu être distingués.

Cependant, ces résultats ne semblent pas remettre en cause l'ancienne présence du pin sylvestre dans les Pyrénées ariégeoises, même si son "ampleur" doit être modulée. En effet, si la fréquence de cette espèce dans les charbonnières de l'Aston ou du Vicdessos avait de quoi surprendre, d'un point de vue bioclimatique elle n'est nullement aberrante. Le pin sylvestre n'apparaît actuellement que rarement sur le versant nord des Pyrénées (de l'Océan au bassin de l'Ariège), mais toujours dans des vallées de la haute chaîne relativement abritées des perturbations atlantiques (haute vallée du Gave de Pau, de la Neste d'Aure, Val d'Aran). Or, les hautes vallées de l'Ariège et du Vicdessos, tout comme les précédentes, présentent un climat océanique d'abri, à tonalité oroméditerranéene, favorable au pin sylvestre (Izard et al., 1985). Son absence actuelle de cette région nous semble ne pouvoir être expliquée que par une action de l'homme plus intense et plus prolongée qu'ailleurs. Le pin sylvestre paraît par exemple plus sensible que le pin à crochets au feux pastoraux (Métailié, 1981). Ce fait semble confirmé par l'absence aujourd'hui encore du pin 
sylvestre (à l'état spontané) dans le Vicdessos où l'impact du pastoralisme a été très important contrairement au massif de l'Aston où subsistent quelques individus agés (Davasse et Galop, 1989). De manière analogue, dana la forêt d'Osséja (Pyrénées orientales), c'est la pression anthropique qui a permis au pin à crochets, plus résistant, d'atteindre vers le bas des surfaces autrefois occupées par le pin sylvestre (Gaussen, 1926).

Si elle n'a pas de valeur statistique ni définitive, cette étude anatomique permet toutefois de rendre compte de la validité de la distinction lorsque la charbonnière se situe dans des conditions stationnelles bien particulières. Par exemple, lorsque pour une charbonnière située à $1400 \mathrm{~m}$ d'altitude, on retrouve des charbons de bois de pin dans un spectre anthracologique dominé par le hêtre, il ne peut s'agir que du pin sylvestre intervenant dans la série du hêtre en pénétrant son étage à la faveur de l'éclaircissement de la forêt montagnarde sous la pression sylvicole. De la même façon, un fragment de pin, trouvé dans une charbonnière au-dessus de $2000 \mathrm{~m}$ d'altitude, sera reconnu comme pin à crochets. Dans ces deux cas, les critères anatomiques précédemment établis s'avèrent pertinents.

Se pose donc le problème du champ d'application des critères de détermination. Deux solutions paraissent possibles. Soit l'on se base sur les critères purement anatomiques (la distinction ne pouvant pas se faire pour l'instant la terminologie sera Pinus sylvestris/uncinata). C'est alors au niveau de l'interprétation écologique que l'on formule des hypothèses. Soit l'on adopte une terminologie différenciée provisoire qui, tout en signalant le problème, permet de hiérarchiser ces premières données dans l'attente de progrès décisifs au niveau xylologique. $C^{\prime}$ est cette dernière solution que nous avons choisi pour une simple raison de clarté des résultats de l'anthracoanalyse, afin aussi de pouvoir revenir plus facilement sur les fragments déjà déterminés. L'emploi du code de dénomination "type" permet cet artifice terminologique. Ainsi, Pinus type sylvestris ou Pinus type uncinata seront employés lorøque les caractères distinctifs précédemment définis sont présents sur tous les cernes annuels, plusieurs étant visibles. Dans tous les autres cas, Pinus sylvestris/uncinata sera la dénomination retenue.

\section{2 - LE SITE CHARBONNE : POSSIBILITES ET DIFFICULTES DE REPRESENTATION DES SUCCESSIONS VEGETALES}

Le site charbonné représente le deuxième niveau d'analyse. Il permet d'appréhender les successions végétales. Ainsi, à l'intérieur d'une charbonnière, les variations, d'un décapage à l'autre, des proportions entre les taxons peuvent-elles théoriquement être interprétées comme des images successives de la végétation forestière. En réalité ce n'est pas si simple. L'aire d'approvisionnement en bois de la charbonnière a, par exemple, pu varier, ce qui introduit le problème des pratiques des charbonniers. La difficulté d'une interprétation fine peut être illustrée par l'étude de deux sites charbonnés, l'un dans la vallée de Soulcem, l'autre dans celle de l'Aston.

A Soulcem 2, cinq charbonnières ont été étudiées et dix-sept taxons mis au jour (Fig. 2). On constate une évolution générale classique : le hêtre est favorisé par l'exploitation forestière au détriment du sapin. Dans le détail pour- 
tant cela se complique. Le cas de la charbonnière 1 est révélateur. Trois phases ont été individualisées dans la couche charbonneuse. La première est caractérisée par la prépondérance du hêtre (42 à $69 \%$ des fragmentø) et la présence du bouleau et des pins. La deuxième est déterminée par la part considérable do aapin (jusqu'à $82 \%$ des fragments). La troisième est une phase de reconquête à hêtre et noisetier. Ces phases avaient été dans un premier temps interprétées comme autant de cycles d'exploitation, du fait de la présence dans le profil de strates stériles (non représentées sur le diagramme anthracologique simplifié cf. Davasse, 1992). Cependant, les datations au radiocarbone ne reconnaissent que deux périodes d'exploitation, l'une au XVIlème siècle, l'autre au XVIlIème siècle, séparées par le colluvionnement présent entre les décapages 4 et 5 . I faut donc prendre en compte, pour la première période, une fluctuation de l'aire de prélèvement. En effet, la synchronie des phases d'exploitation du hêtre et du sapin ne s'explique que si l'aire de prélèvement est différente. Ce qui suppose une forêt gérée. Or, dans les Pyrénées, la mise en défens des sapinières (bois de réserve) pour le bois d'oeuvre est la forme principale de gestion sylvicole par la société locale. Les résultats obtenus peuvent donc donner lieu à deux interprétations. Il s'agirait soit d'une exploitation différentielle où les fûts de sapin sont abattus pour le bois d'oeuvre et les branchages utilisés pour les charbonnières, soit $d^{\prime} u n$ charbonnage radical d'une sapiniè̀re - on se situe en effet à une période charnière où, sous l'effet d'une pression accrue, les délits dans les mises en défens deviennent courants (Davasse et Galop, 1990). Quoi qu'il en soit, les sapinières de réserve disparaissent à la fin du XVIIIème siècle comme le montre les données de la dernière période.

Le second exemple se situe dans la vallée de l'Aston, entre 1300 et $1700 \mathrm{~m}$ (Aston 2). Jusqu'à $1550 \mathrm{~m}$ d'altitude, ce versant est recouvert d'une chênaie ( $Q$. petraea Liebl.), surmontée par le hêtre. Au-dessus, vers $1650 \mathrm{~m}$, se trouvent quelques bouquets de pins à crochets. La présence massive du chêne à ces altitudes est une entorse au modèle bioclimatique (Gaussen, 1926 ; Dupias, 1985), et actuellement la régénération de cette essence reste hypothétique tandis que celle du hêtre est remarquable. Il faut donc aller chercher une explication dans le passé de la végétation. Mais les textes et la palynologie donnent dea explications contradictoires. D'après les archives forestières, à la fin du XVIIème siècle, ce versant est planté de hêtre et de sapin, un siècle plus tard de chêne ct de noisetier ( ${ }^{l}$ ). Les données polliniques, provenant certes de sondages éloignés du site, ne sont guère explicites. Elles font état d'une forte représentation da chêne en début de séquence, représentation qui diminue par la suite de façon constante (Galop, 1992). En l'absence de datation au Carbone 14 (qui sont en courg), le diagramme anthracologique de Aston 2 ne permet pas de conclusion définitive (Fig. 3). Mais, infirmant les textes, il indique d'ores et déjà que $k$ chêne et le sapin ont cohabité. Par contre, sans chronologie, la dynamique progressive du hêtre par rapport au chêne dans la charbonnière 2 ne peut pas être interprétée, le replat oe trouvant actuellement dans la chênaie.

Ainsi dans le cas de l'étude des succession végétales à l'échelle du site, la complexité des processus demande un approfondissement de la recherche, en s'orientant peut-être vers une reconstitution de charbonnage sur un site déjà reconnu. La chronologie donnée par le radiocarbone est donc indispensable à 


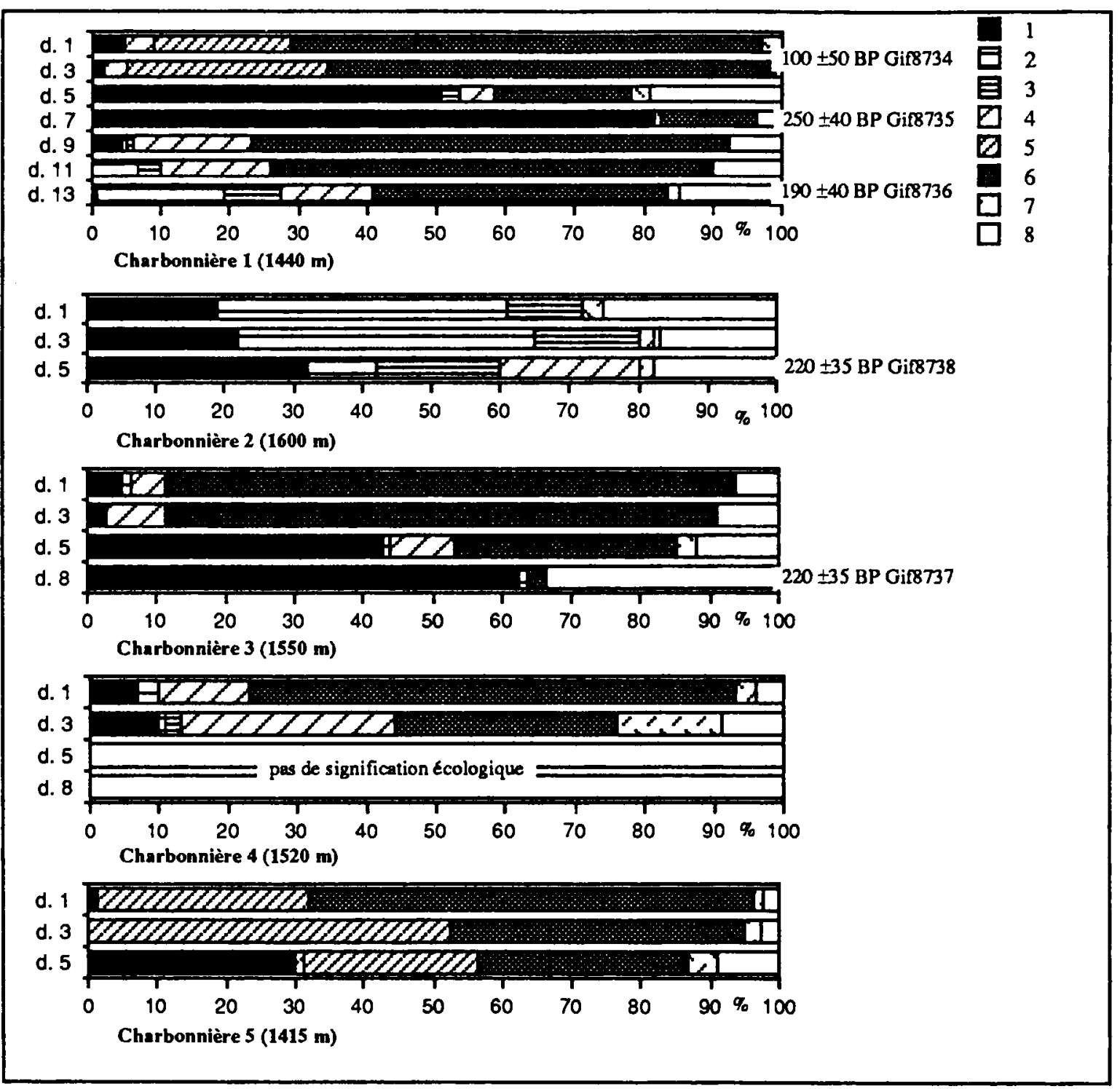

Fig. 2.- Diagramme anthracologique de Soulcem 2 (Tignalbu). 1 - Abies alba 2 - Pinus type sylvestris. 3 Pinus typo uncinata 4 - Betula. 5 - Corylus avellana 6 - Fagus sylvatica. 7 - Varia. 8 - Indéterminable.

Fig. 2.- Charcoal diagram of Soukem 2 (Tignalbu). 1 - Abies alba. 2 - Pinus sylvestris. 3 - Pinus uncinata. 4 Betuia 5 - Corylus aveliana. 6 - Fagus sylvatica. 7 - Varia. 8 - Undetermined.

l'interprétation des résultats $\left({ }^{2}\right)$, et ce malgré une marge d'incertitude statistique qui peut paraître importante pour des périodes historiques. Cette "imprécision" des datations se remarque généralement par rapport aux informations du mobilier archéologique ou des textes historiques. Or, pour les Pyrénées, il n'existe pas de documents exploitables avant le XVIIème siècle en ce qui concerne les espaces forestiers. Quant aux charbonnières, elles ne recèlent aucun mobilier susceptible de les dater.

\section{3 - LA VALLEE : L'EVOLUTION DE L'ESPACE FORESTIER}

Ce troisième niveau d'étude permet de suivre l'évolution de l'espace forestier d'une vallée. Seules sont retenues les grandes essences forestières pyrénéennes : chênes, hêtre, sapin, pin sylvestre, pin à crochets. En prenant en compte les conditions topographiques (qui déterminent le dénivelé drainé par le

(2) L'ensemble des datations sont effectuées, sous la direction de M. Fontugne, par le Centre des Faibles Radioactivités du CNRS à Git-sur-Yvette. 


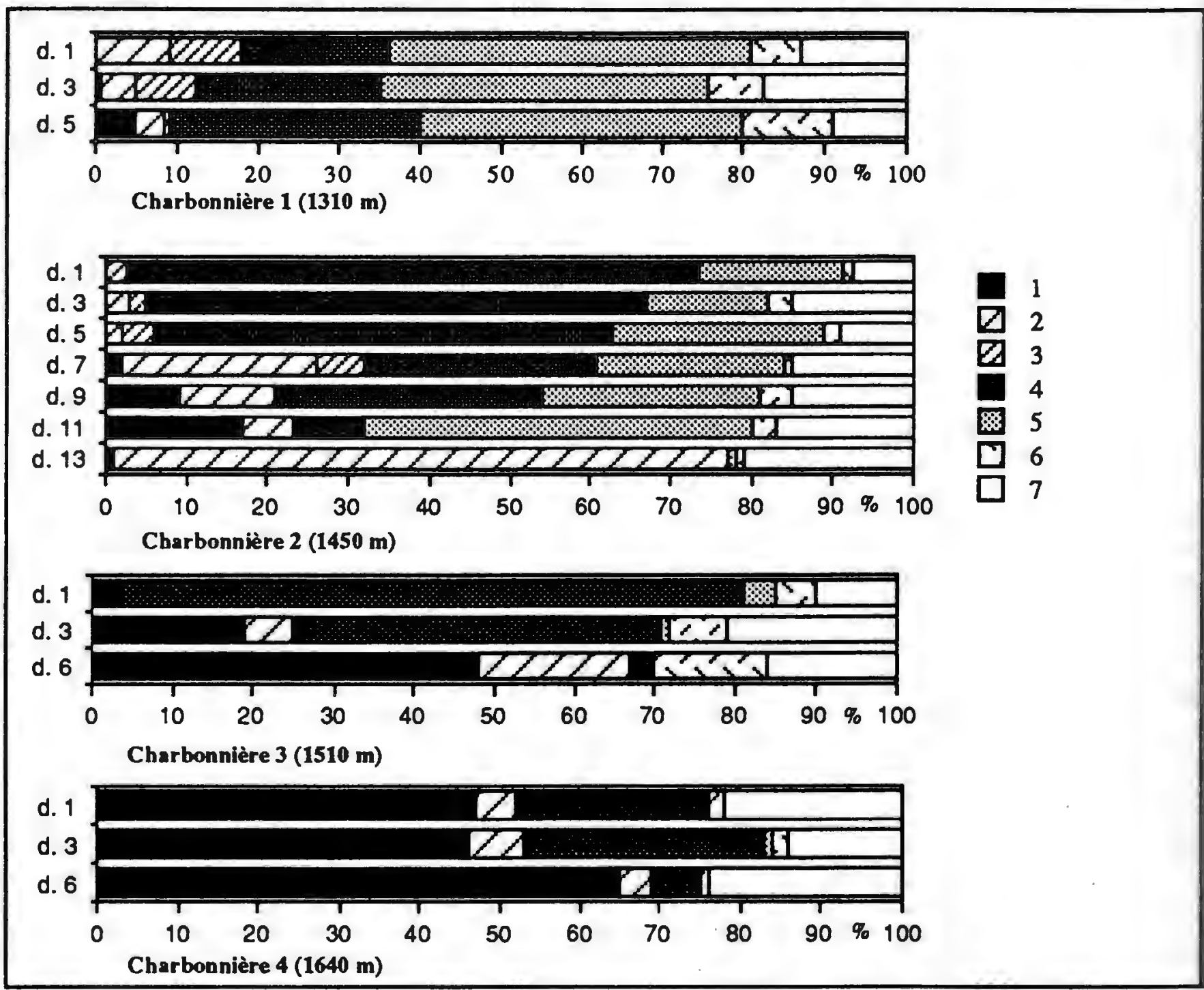

Fig. 3 - Diagramme anthracologique de Aston 2 (bols de l'Agrbou). 1 - Abies alba. 2 - Betula. 3 - Contu avellana. 4 - Fagus sylvatica 5 - Quercus à f. c. 6 - Varla. 7 - Indéterminable.

Fig. 3.- Charcoal diagram of Aston 2. 1 - Abies alba. 2 - Betula. 3 - Corylus avellana. 4 - Fagus sylvatica 5 Quercus. 6 - Varia. 7 - Undetermined.

lançage du bois jusqu'au replat), on peut tracer l'ancienne limite supérieure de la forêt, variant au-dessus de celle des charbonnières. En notant les discordances dans la répartition des charbonnières (par exemple l'absence de place de charbonnage sur des surfaces planes propices à l'élevage), on peut proposer une interprétation des modalités d'occupation de l'espace forestier : défrichoments à des fins pastorales ou charbonnage. L'analyse de la distribution spatiale des charbonnières permet de localiser l'ancienne forêt charbonnée. Enfin, l'étude de sites charbonnés échelonnés selon un gradient altitudinal donne un schéma évolutif de l'étagement de la végétation forestière.

L'exemple de la vallée de Soulcem dans le Vicdessos confirme la validité de cette démarche. Actuellement, la limite supérieure de la forêt y est particulièrement basse, ne dépassant pas $1200-1400 \mathrm{~m}$. Mais des replats de charbonnage se rencontrent jusqu'à $2150 \mathrm{~m}$, recouvrant dans ce cas la limite supérieure potentielle du pin à crochets (Fig. 4). Seuls les versants qui subiesent de fortes contraintes géomorphologiques et les ombilics glaciaires précocoment déboisés - avant 3500 BP comme l'attestent les données de la palynologie (Jalut et al., 1984) - ne portent pas de charbonnières.

Dans cette vallée, trois sites charbonnés ont été étudiés. Ils s'échelonnent entre 1400 à 2000 mètres d'altitude. En ne retenant que les espèces fores- 
tières précitées, le recours aux sources écrites et aux datations au Carbone 14 permet d'envisager un modèle cartographique de l'évolution des grands ensembles forestiers. L'analyse de l'espace montagnard actuel (le seul connu !) constitue le point de départ, les différentes informations disponibles, de moins en moins fiables au fur et à mesure que l'on remonte le temps, viennent, par étapes successives, modifier cette image initiale (méthode régressive). Le modèle ainsi obtenu est assez théorique. Il autorise cependant une première généralisation des résultats (Fig. 5).

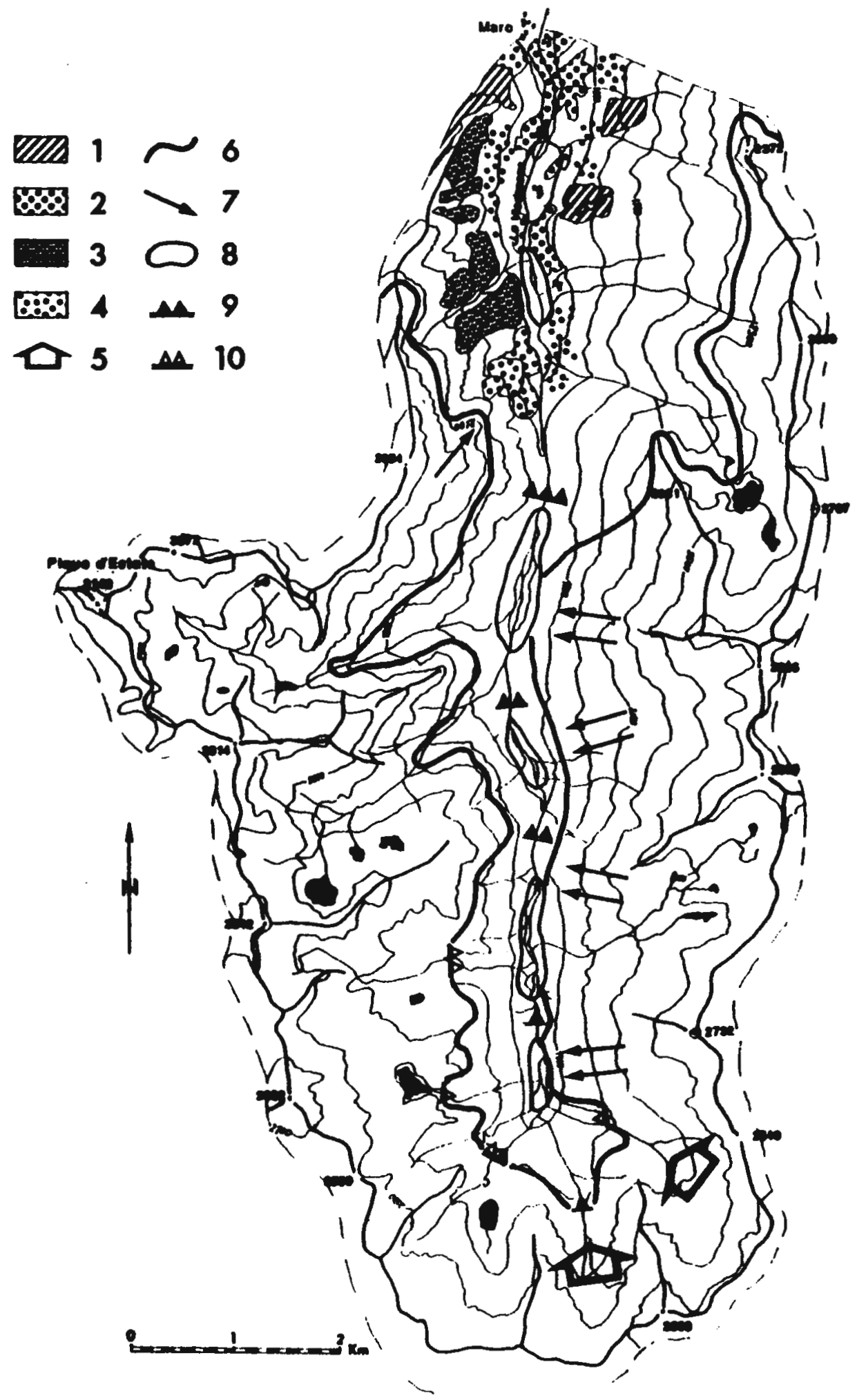

Fig. 4.- La foret actuelle et l'ancienne limite superieure de la forét de Soulcem.

1 - Plantations XIXèmeXXème siécie. 2 - Hotrale-chenaie. 3 - Boisements clairs on milieu contraignant (Quercus petraea, Betula pendula, Fagus syvatica). 4 Extension récente à bouleau, sureau ou saule. 5 - Effet de foehn. 6 - Limite supérieure de la foret extrapolée d'après la répartition des charbonnieres. 7 - Contraintes morphoclimatiques. 8 $\mathrm{Pla}$ de fond de vallée (absence de charbonnières). 9 - Verrou de vallée glaciaire. 10 Verrou de sortie de cirque glaciaire.

Fig. 4.- The present forest and the past timber-line of Soukem valley.

1 - Coniferous plantations 19th-20th centuries. 2 -

Declduous forest (beech and oak). 3 Cleared forest (Betula pendula, Quercus petraea, Fagus sylvatica). 4 - Reafforestation (Betula Salix, Sambucus racemosa). 5 - Foohn. 6 - Past timber-line based on the distribution of charcoal kilns. 7 . Morphocllmatic processes. 8 - Glacial ombllic : pastored land from 3500 BP. 9 - Construction of glaclal valioy. 10 - Construction of glacial cirque. 


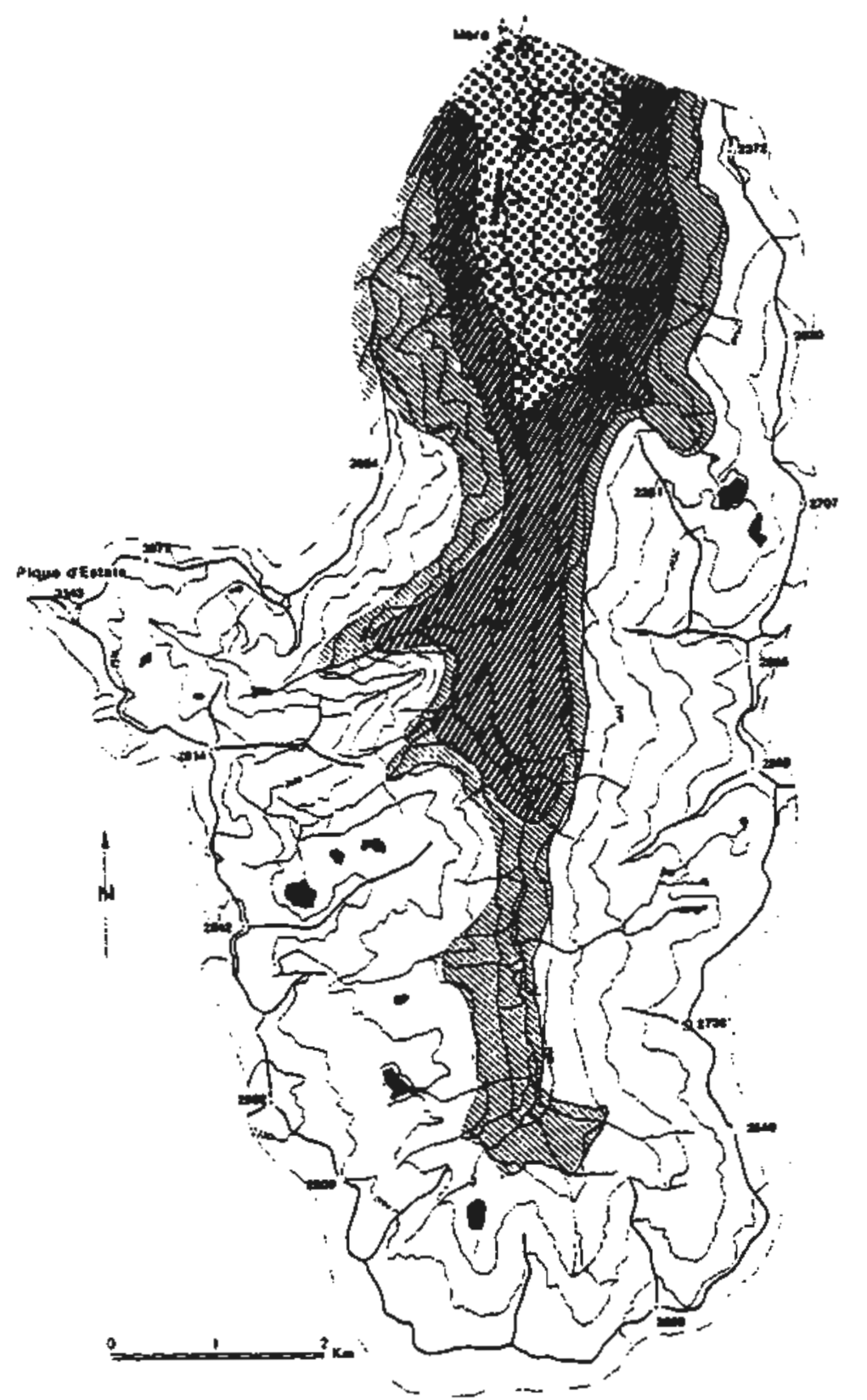

A

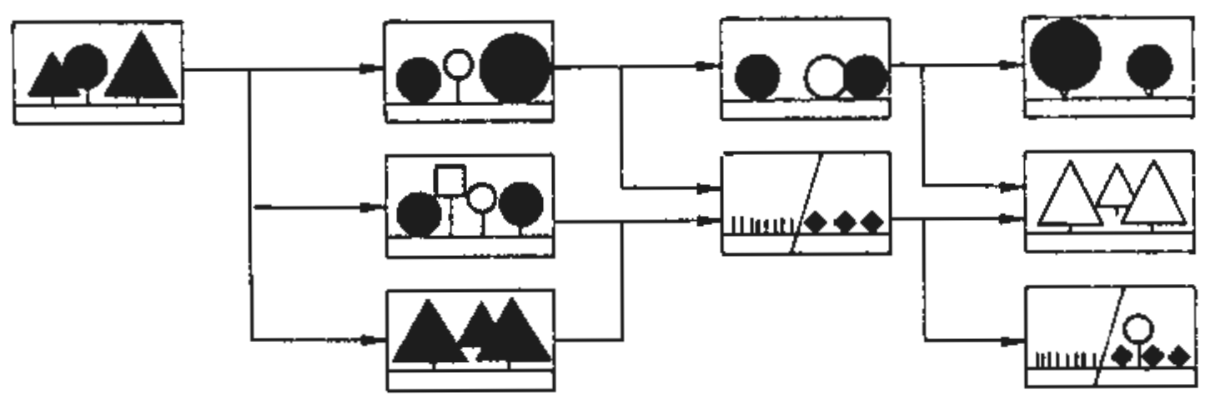

B
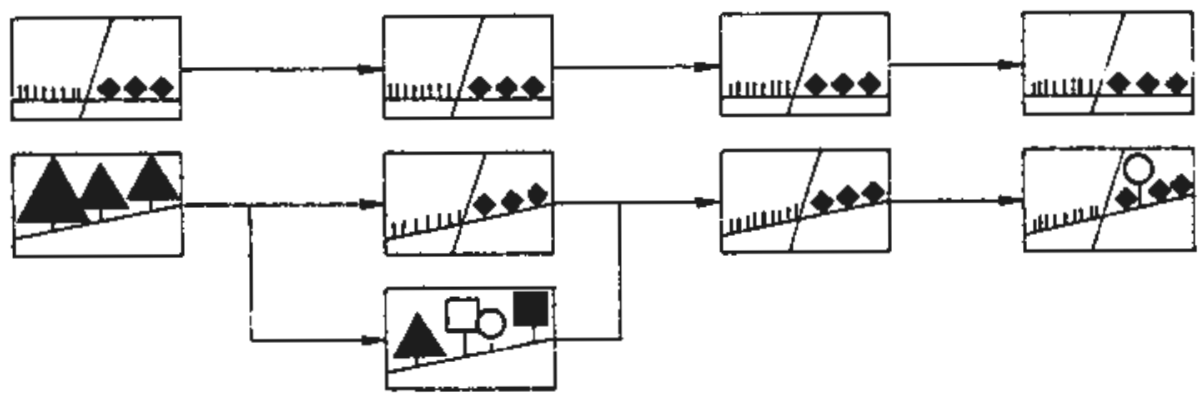

C
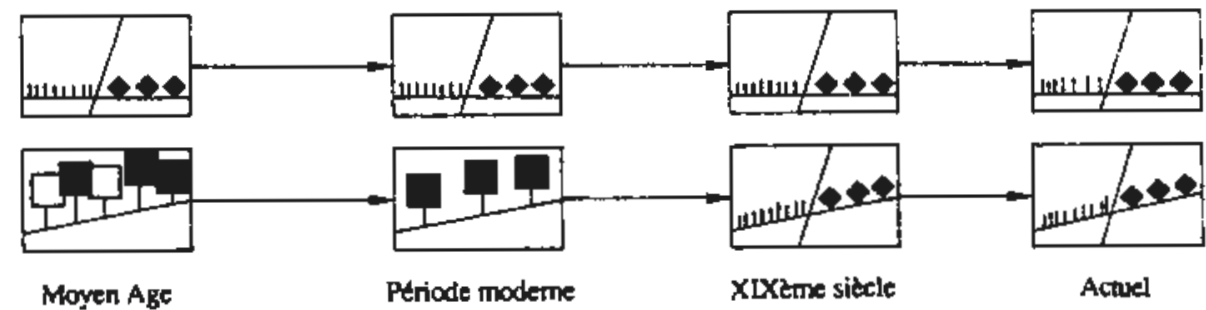

Moyen Age

Période modetne

XLXene sitzele

Actuel

Wone A

zone B

zone $C$

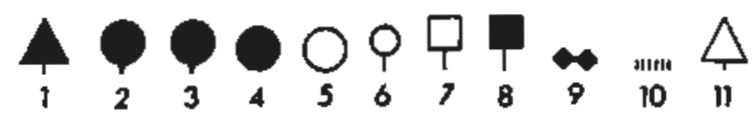

Flg. 5. - Modele cartographique d'évolution des espaces forestlers. 1 - sapin. 2 - hetre (futaie). 3 - hatre (tallis). 4 - hetre (dutale sur souche). 5 - nolsetler (fourro). 6 - bouleau. 7 - pin sylvestre. 8 - pin a crochets. 9 - lande. 10 - pelouse. 11 - plantation.

Fig. 5.- Cartographlc model of torested areas evolution. 1 - slver fir (high trees). 2 - beech (high trees). 3 - beech (copplice). 4 - beech (copplce

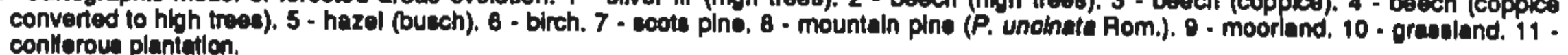


Trois grandes zones, et autant de seuils biogéographiques, ont été différenciés. La première s'étend jusqu'à $1600 \mathrm{~m}$ environ (zone A). A partir de la sapinière-hêtraie du Moyen Age, la forêt évolue différemment selon la gestion humaine : soit vers un taillis de hêtre à bouleau et pin sylvestre, utilisé pour l'affouage de la communauté et des forges ; soit vers une sapinière, dans les secteurs mis en défens pour le bois d'œuvre. Au maximum de pression anthropique, au XIXème siècle, le taillis de hêtre à noisetier reste le seul peuplement forestier. Aujourd'hui, après un quasi-abandon de l'espace, alternent futaies sur souche de hêtre, plantations de la fin du XIXème siècle et espèces pionnières sur landes. Entre 1600 et $1800 \mathrm{~m}$ (zone B), les plas qui ont fait l'objet de défrichements pastoraux dès l'Age du Bronze sont asylvatiques tout au long de la période étudiée. Sur les versants, l'ancienne sapinière charbonnée a été progressivement pénétrée par les pins et le bouleau, voire par endroit précocement éradiquée, tant et si bien qu'au XIXème siècle tout peuplement forestier a disparu. Un processus sensiblement identique s'est produit dans la zone C. Vers le bas des versants, le pin sylvestre s'efface au profit du pin à crochets dont les peuplements s'éclaircissent, puis disparaissent. Vers le haut, la pinède à pin à crochets est anéantie avant le XIXème.

Une série de huit datations au Carbone 14 fait remonter le maximum de l'exploitation par le charbonnage dans la vallée de Soulcem à la période moderne, les dernières étapes se prolongeant jusqu'au XVIIIème siècle. C'est là une donnée nouvelle. Ni les diagrammes palynologiques, ni les textes ne permettaient d'envisager dans cette vallée une exploitation de cette importance aussi tardive. On peut cependant mettre cet élément en corrélation avec une vague d'installations de "moulins à fer" au cours du XVIème siècle $\left(^{3}\right)$. Cela confirme que la crise forestière du Vicdessos a été plus tardive que l'historiographie locale ne le supposait (Davasse et Galop, 1990). En effet, de nombreux auteurs ont décrit le déboisement du bassin du Vicdessos comme un processus ancien, bien antérieur à la réformation de Froidour (1669) $\left({ }^{4}\right)$ qui ne fait pas état, entre autres, de la forêt subalpine. L'étude des charbonnières apporte là un élément de premier ordre en précisant que l'ultime exploitation des espaces forestiers d'altitude s'est située au XVIIIème siècle.

Ce premier bilan de l'étude des espaces forestiers charbonnés dans la moitié orientale des Pyrénées est riche d'enseignements. Outre le fait qu'elle apporte une définition spatiale essentielle (localisation des anciens peuplements forestiers, variation de la limite supérieure de la forêt, mode d'occupation de l'espace, etc.), l'étude des charbonnières permet, à l'aide des datations au Carbone 14, de rendre compte des processus dans la durée. Elle vient alors pallier avec succès aux manques des sources écrites ou des données palynologiques. A une échelle plus grande, le bilan doit cependant être nuancé. Un approfondissement s'impose en ce qui concerne la mise en évidence des successions végétales sur le site charbonné. Pour cela de nombreuses datations au C14 sont nécessaires, elles permettent de relier les données anthracologiques à celles des sources écrites et des séquences palynologiques. Ce n'est qu'à ces conditions que l'on peut envisager des scénarios d'évolution des espaces forestiers.

(3) Manuscrit 638, Bibliothèque municipale de Toulouse ; série E6, Archives départementales de l'Ariège (communication Jean Cantelaubo).

(4) 2B31, Archives départementales de PArlège. 


\section{BIBLIOGRAPHIE}

BONHOTE J, et J.L. VERNET, 1988,- La "mémoire des charbonniéres". Essal de reconstitutlon des milleux forestlers dans une vallée marquée par la métallurgie (Aston, Haute Ariege). Aev. Forestlere Françalse, 40 (3), 197-212.

DAVASSE B., 1992.- Aspects máthodologiques de lanthracoanalyse des charbonnieres. Histoire des forets de la vallée de Soulcem (Vlcdessos, Pyrénées arlégeoises). Cahlers de I"lsard, 3, 205-219. [Actes du Colloque "Proto-Industries et Histoire des forets", 11-12 octobre 1990, Fobx].

DAVASSE B. $\theta$ I D. GALOP, 1969.- Le charbon de bols et le pollen : éléments pour une approche de lévolution historique du paysage forestler dans les Pyrénées arlégeoises. Acta Blologica Montana, 9, 333340. [Actes du Colloque "Ecosystemes d'altitude", novembre 1989, Pau].

DAVASSE B. et D. GALOP, 1990.- Le paysage forestler du Haut Vlodessos : 6volutlon d'un milleu anthroplse. Rev. Gégraphique des Pyrónés ot du Sud-Ouest, 61 (4), 433-457.

DAVASSE B. et D. GALOP.- Impact des activités pastorale et motaliurgique sur les fortsts dahlitude des Pyrénés ariegeolses (France). Actes du Colloque Intemational "Ecologle ot Blogeographle apines", septembre 1990, La Thulle (thalle), 8 p., sous presse.

DUPIAS G., 1985.- Végétation des Pyrénées. Notce détaillé de la partle pyrénéenne des feuilles 69 Bayonno, 70 Tarbes, 71 Toulouse, 72 Carcassonne, 76 Luz, 77 Foxx, 78 Perplgnan. CNRS (Ed.), Parts, 209 p.

GALOP D., 1992.- Approche palynologique de rimpact de la métallurgle au bois sur les forts de la vallé du Quloulés (Pyrénées arlégeolses, France). Cahiers de risard, 3, 257-270. [Actes du Colloque "Proto-industrles ot Histolro des forets", 11-12 octobre 1990, Folx].

GAUSSEN H., 1926.- Vegétatlon de la molité orentale des Pyrénés : sol, climat, végétation. Documents pour une carte de la végétation. Lechevaler (Ed.), Paris, 560 p., 2 cartes h.t.

HEINZ C., E. BADAL GARCIA, I. FIGUEIRAL, E. GRAU ALMERO, S. THIEBAULT ot J.L. VERNET, 1990.Identificatlon des charbons de bols prehistoriques méditerraneens, chronologie et répartition. $\mathcal{L}$ European Study Group on Physical, Chemical, Mathematcal and Blological Techniques Appliad to Archaelogy, 22 , 161-172. [Wood and Archaeology Conference, October 2nd-3rd 1987, Louvain].

ZAAD V.- L'art du charbonnler. Contributlon ethnobotanique et géographico-historlque à létude des paysages metallurgiques d'aprés l'anthracoanalyse des charbonnleres. Actes du colloque tos charbons de bots, les anclens tcosystemos of be role de thomme", Montpelller, 10-12 septembre 1991. 13 p., a paralitio.

ZARD M., H. CASANOVA, B. DEVAU et G. PAUTOU, 1985.- Continentaltí et notlon de zone inteme dans las Pyrónés. Acta CEcologicalCEcologica Generalls, 6 (4), 317-344.

JACOULT C., (1955), Atlas d'anatomle des bols des conlières. Centro Technique du Bols (Ed.), Parls, 2 vol

JALUT G., A. ESTEBAN AMAT, P. PAGES ot M. MARDONES, 1984.- Queiques aspects de laction do Thomme sur le milieu montagnard pyrénên : consequences phytogéographiques. Documents dEcologle Pyrensenne, 3-4, 503-509. [Actes du colloque international "Ecologie et Biogégraphle des Milleux Montagnards of de Haute Attide", Gabas, 10-12 septembre 1982].

METAILIE J. P., 1981.- Le feu pastoral dans les Pyrénées contrales (Barousse, Oueill, Larboust). CNAS (Ed) Toulouse, 294 p, 2 cartes h.t. 